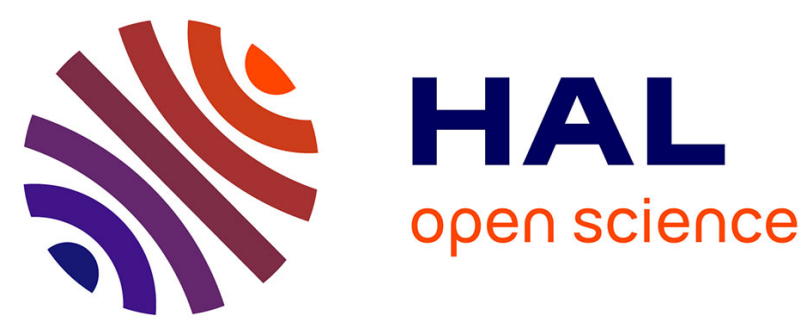

\title{
L'APPROPRIATION DE LA RSE PAR LES \\ DIRIGEANTS DE PME. LE RÉSEAU COMME VECTEUR DE L'APPRENTISSAGE MANAGÉRIAL
}

Elise Bonneveux, Jean-Yves Saulquin

\section{- To cite this version:}

Elise Bonneveux, Jean-Yves Saulquin. L'APPROPRIATION DE LA RSE PAR LES DIRIGEANTS DE PME. LE RÉSEAU COMME VECTEUR DE L'APPRENTISSAGE MANAGÉRIAL. Revue management \& avenir, 2009, 10.3917/mav.023.0170 . hal-02429187

\section{HAL Id: hal-02429187 \\ https://hal.science/hal-02429187}

Submitted on 6 Jan 2020

HAL is a multi-disciplinary open access archive for the deposit and dissemination of scientific research documents, whether they are published or not. The documents may come from teaching and research institutions in France or abroad, or from public or private research centers.
L'archive ouverte pluridisciplinaire HAL, est destinée au dépôt et à la diffusion de documents scientifiques de niveau recherche, publiés ou non, émanant des établissements d'enseignement et de recherche français ou étrangers, des laboratoires publics ou privés. 


\title{
L'APPROPRIATION DE LA RSE PAR LES DIRIGEANTS DE PME. LE RÉSEAU COMME VECTEUR DE L'APPRENTISSAGE MANAGÉRIAL
}

\author{
Elise Bonneveux et Jean-Yves Saulquin
}

Management Prospective Ed. | « Management \& Avenir »

2009/3 n² $23 \mid$ pages 170 à 186

ISSN 1768-5958

Article disponible en ligne à l'adresse :

https://www.cairn.info/revue-management-et-avenir-2009-3-page-170.htm

Distribution électronique Cairn.info pour Management Prospective Ed..

(C) Management Prospective Ed.. Tous droits réservés pour tous pays.

La reproduction ou représentation de cet article, notamment par photocopie, n'est autorisée que dans les limites des conditions générales d'utilisation du site ou, le cas échéant, des conditions générales de la licence souscrite par votre établissement. Toute autre reproduction ou représentation, en tout ou partie, sous quelque forme et de quelque manière que ce soit, est interdite sauf accord préalable et écrit de l'éditeur, en dehors des cas prévus par la législation en vigueur en France. Il est précisé que son stockage dans une base de données est également interdit. 


\title{
L'appropriation de la RSE par les dirigeants de PME. Le réseau comme vecteur de l'apprentissage managérial
}

\author{
par Elise Bonneveux et Jean-Yves Saulquin
}

\section{Résumé}

Cet article vise à s'interroger sur la manière dont les dirigeants de PME peuvents'approprier le concept de RSE et décident de l'opérationnaliser grâce au réseau d'entreprises. Dans une première partie, les auteurs reviennent sur la théorie des parties prenantes, vue comme un moyen d'expliquer la posture du dirigeant face aux enjeux de la responsabilité sociale de l'entreprise. Si cette théorie permet de décrire les parties prenantes et leurs attentes, elle ne permet pas vraiment d'expliquer pourquoi et comment le dirigeant s'implique. Parmi les nombreux déterminants possibles de l'action responsable, les auteurs décrivent dans une deuxième partie le rôle que peut jouer le réseau dans l'apprentissage managérial. La troisième partie permet d'illustrer cette réflexion en restituant l'exemple d'un groupement de PME d'Indre et Loire qui exerce une influence prépondérante dans la mise en place de pratiques socialement responsables chez ses adhérents. L'apprentissage réticulaire est présenté dans cet article comme le vecteur principal de l'action managériale dans le domaine de la RSE.

\section{Abstract}

This article examines the way in which SME managers may adopt the CSR concept and decide to put it to practice using the support of the corporate network. In the first part, the authors review the stakeholder theory, seen as a means of explaining the positioning of the manager faced with the challenges of corporate social responsibility. Where this theory helps to describe the stakeholders and how management envisages responding to the pressures they exert, it does not help to explain why and how management becomes involved. Of the numerous possible factors determining the action of responsibility, in the second part of the article, the authors describe the role that the network can play in the management learning process. The third part helps to illustrate this discussion by showing the example of a grouping of SME's in the Indre et Loire region of France who exert considerable influence on their members to implement practices of social responsibility. The network of learning is presented in this article as the principal vehicle for enabling management to take action in the field of CSR.

De nombreux articles ont montré combien il était difficile pour des dirigeants de PME de s'informer et d'innover en matière de RSE. Les manques de connaissance, 


\section{L'appropriation de la RSE par les dirigeants de PME. Le réseau comme vecteur de l'apprentissage managérial}

de temps et d'information sont souvent des freins majeurs au développement des pratiques responsables (Berger-Douce, 2008). Les PME qui s'engagent dans une telle stratégie affirment néanmoins que les retombées sont souvent bénéfiques et concernent aussi bien la réduction des coûts, l'augmentation de la fidélité des salariés, de meilleures relations avec les instances territoriales, l'acquisition de nouvelles connaissances ainsi qu'une meilleure réputation (Luetkenhorst, 2004).

Cet article vise à s'interroger sur la manière dont les dirigeants de PME s'approprient le concept de RSE et décident ensuite de l'opérationnaliser. $\mathrm{Ce}$ processus d'apprentissage peut notamment être initié par l'appartenance à un réseau d'entreprises.

Dans une première partie, les auteurs reviennent sur la théorie des parties prenantes et ses apports. Cette grille de lecture théorique permet d'expliquer la posture du dirigeant face aux enjeux de la responsabilité sociale de l'entreprise. L'intérêt de ce modèle contractuel partenarial réside dans la description des enjeux sociétaux en mettant en évidence les parties prenantes (quels sont les partenaires concernés) et leurs attentes (quelles sont les pressions exercées). Mais cette approche est limitée dans la mesure où elle ne permet pas vraiment de comprendre les fondements de la RSE, son processus d'appropriation et la méthode de gouvernance pour les parties prenantes (quelle est la dynamique de l'implication).

Parmi les nombreux déterminants possibles de l'action responsable, les auteurs décrivent dans une deuxième partie le rôle essentiel que peut jouer le réseau d'entreprise dans l'apprentissage managérial face aux enjeux de la RSE. II s'agit d'un facteur déterminant au même titre que la formation, l'âge ou les valeurs et qui est peu abordé dans les travaux de recherche. L'accent sera mis sur le rôle de sensibilisation, d'accompagnement et de coordination qu'il permet. La vision du dirigeant s'en trouve modifiée et favorise l'action innovante.

La troisième partie permet d'illustrer cette réflexion en restituant l'exemple d'un groupement de PME d'Indre et Loire (le GEIDA) qui exerce une influence prépondérante dans la mise en place de pratiques socialement responsables chez ses adhérents. L'apprentissage réticulaire est présenté ici comme le vecteur principal de l'action managériale dans le domaine de la RSE.

\section{La théorie des parties prenantes pour comprendre la posture du dirigeant face à la RSE}

Dans cette partie, nous reviendrons sur les apports de la théorie des parties prenantes mais aussi sur ses limites. Pour ce faire, nous nous interrogerons sur la manière dont le dirigeant de PME perçoit la RSE et sur la pertinence de la 
théorie des parties prenantes en tant que grille de lecture des comportements managériaux.

\subsection{L'éclairage théorique proposé par la théorie des parties prenantes pour appréhender la démarche RSE du dirigeant}

Bowen (1953) présente la RSE comme une obligation faite aux dirigeants de mettre en œuvre des stratégies, de prendre des décisions, et de garantir des pratiques qui soient compatibles avec les objectifs et les valeurs de la communauté en général. Cette approche a été complétée par les travaux plus formels de Caroll (1979) qui propose un modèle conceptuel reposant sur trois dimensions : les principes de RSE, la manière dont l'entreprise met ses principes en pratique (sa sensibilité sociale), et les valeurs sociétales qu'elle porte. Wartick et Cochran (1985) complètent cette approche en spécifiant que la RSE résulte de l'interaction de trois dimensions : les principes, les processus et les politiques. Ils ajoutent que la RSE est une approche microéconomique de la relation entre l'entreprise et son environnement. En 2001, la Commission des Communautés Européennes définit la RSE comme « l'intégration volontaire par les entreprises de préoccupations sociales et environnementales à leurs activités commerciales et leurs relations avec leurs parties prenantes ».

Pour Allouche et Alii (2004), adopter un comportement de responsabilité sociale " c'est répondre à la nécessité de maximiser les objectifs de l'entreprise par l'entremise de sa rentabilité, au profit toujours de l'actionnaire, mais aussi de ses autres partenaires $"$.

La satisfaction des parties prenantes apparait comme un des facteurs déterminants dans une démarche RSE. La RSE se présente comme une vraie problématique managériale sous la pression des parties prenantes. II faut créer de la valeur par la cohésion des acteurs et c'est le jeu des acteurs qui permet de repérer ceux qui seront prédominants. Un consensus émerge des différentes approches académiques et pratiques de la RSE pour lui conférer trois piliers : environnemental, économique et social (ou sociétal). La RSE est un construit multidimensionnel qui se présente comme un ensemble de paramètres complémentaires qui imposent des arbitrages et qui amènent le manager à décider de priorités en fonction de contraintes ou de choix politiques.

Il est donc difficile de parler de la RSE sans traiter la question des décisions qui la fondent. Voilà pourquoi il manque un quatrième pilier à la représentation de la RSE, celui de la gouvernance. C'est ce pilier qui englobe les décideurs et les processus de décision et qui permet de comprendre quelle place l'entreprise réserve à ses parties prenantes (Igalens, 2009).

L'idée que le manager doit prendre en compte les attentes de différentes parties prenantes et pas seulement celles des actionnaires n'est pas nouvelle. Abrams 


\section{L'appropriation de la RSE par les dirigeants de PME. Le réseau comme vecteur de l'apprentissage managérial}

(1951) définit la profession de dirigeant comme celui qui doit conduire les affaires de manière à maintenir un équilibre équitable entre les revendications des différents groupes directement intéressés. Bowen (1953) modélise l'entreprise comme un centre dont l'influence se propage dans des cercles de plus en plus grands. Le premier cercle contient les employés, puis viennent les actionnaires, les consommateurs, les fournisseurs, la communauté, les concurrents. Enfin, le cercle extérieur représente le public dans son ensemble.

Cette notion de stakeholder (littéralement : un détenteur d'enjeu) a été approfondie par Freeman (1984) qui le définit comme un « individu ou groupe d'individus qui peut affecter ou être affecté par la réalisation des objectifs organisationnels $"$. Cette nouvelle représentation de l'entreprise vue au centre d'une roue et reliée à ses stakeholders par des rayons incite le manager à repenser la stratégie en fonction des parties prenantes.

Ce modèle des parties prenantes se justifie par un contexte libéral de réduction du rôle de l'Etat (les entreprises devant prendre le relais), mais aussi par des questions d'altruisme et de morale. Baret (2007) rappelle « qu'à l'instar de Jones et Wicks (1999), il est possible de synthétiser les valeurs du stakeholder model par le rejet de la seule maximisation du profit, l'idée selon laquelle aucune entreprise ne peut perdurer si elle sert uniquement ses intérêts propres, la compatibilité entre capitalisme et moralité (...) et enfin, l'importance de la justice distributive et de l'équité ".

Damak-Ayadi et Pesqueux (2003) proposent une synthèse des différents courants théoriques relatifs aux parties prenantes. Le courant descriptif considère que l'organisation est au centre de coopérations et de compétitions possédant chacune leur valeur intrinsèque. Le courant instrumental repose sur l'idée selon laquelle les entreprises qui gèrent leurs parties prenantes sont plus performantes en termes de profitabilité, de stabilité, et de croissance. Enfin, la théorie éthique normative insiste sur les obligations éthiques de la firme et sur les manières de les assumer, sans pour autant nier l'intérêt qu'elle porte à ses objectifs de performance économique.

Cette théorie des parties prenantes a fait l'objet de travaux hétérogènes et de nombreuses typologies, qui se complètent plus qu'elles ne s'opposent. Elles sont proposées afin d'identifier les différentes parties prenantes et leurs poids respectifs (Gond et Mercier, 2005).

Ces typologies reposent sur la nature (interne ou externe) des parties prenantes, leurs intérêts, leurs contributions, les relations volontaires ou involontaires avec la firme (Clarkson, 1985). Ainsi, pour répondre à la question 'comment certaines parties deviennent prenantes ?' Mitchell, Agle et Wood (1997) les classent à partir de trois attributs : le pouvoir (pour les groupes d'acteurs qui ont la capacité d'influencer les décisions actuelles de la firme), la légitimité (quand un groupe 
est socialement reconnu et accepté), et l'urgence (quand les parties prenantes demandent une attention immédiate face à une situation critique d'exposition à un risque). Ces auteurs proposent ainsi d'établir une hiérarchie entre les parties prenantes qui, dans la pratique, ne sont pas sur un même pied d'égalité.

Pour l'action managériale, la satisfaction des parties prenantes reste un facteur déterminant pour le choix et la mise en œuvre des pratiques de RSE. Dans cette optique, nous allons essayer d'identifier quels peuvent être les comportementstypes du dirigeant en matière de RSE.

\subsection{Les postures managériales face à la RSE et les limites explicatives de la théorie des parties prenantes}

Pour comprendre les comportements managériaux en matière de RSE, Saulquin et Schier $(2005,2007)$ ont avancé l'idée selon laquelle les dirigeants définissent différemment la RSE suivant leur degré d'ouverture (vision managériale fermée ou ouverte de la firme) et leur approche de la performance (approche statique ou dynamique). Ils définissent une typologie des perceptions managériales de la RSE qui peut être perçue comme :

- Une contrainte supplémentaire de type normatif. Dans ce cas, il existe un risque d'instrumentalisation de la RSE qui ne procure aucun effet de levier managérial. Le manager cherche avant tout à apporter une réponse précise à chaque « critère RSE ».

- Un levier d'ouverture de l'entreprise. La RSE est analysée comme un moyen de mieux gérer les relations avec les autres parties prenantes et notamment la communauté. La RSE apparaît alors comme un vecteur de communication opportuniste. Ces démarches s'apparentent à une approche " cosmétique » de la RSE qui permet néanmoins de redéfinir les rapports avec les parties prenantes.

- Un levier de dynamique interne. La RSE, en faisant prendre conscience aux différentes parties prenantes de la nécessité de changer certaines pratiques, peut devenir un levier d'innovation. Dans cette optique, l'accent est mis sur les processus et les résultats attendus à moyen terme. La firme cherche à faire évoluer en profondeur ses pratiques et la RSE permet par exemple d'y associer les salariés, de lancer des chantiers (certification par exemple).

- Un levier stratégique où la RSE oblige l'entreprise à procéder à une relecture critique de sa vocation et de son mode de fonctionnement (son business model). Selon Bowen (1953), la RSE au sens strict ne devrait correspondre qu'à cette dernière vision. II ne s'agit plus de répondre de manière fragmentée aux " exigences » de la RSE, mais de proposer une vision fédératrice et sociétale de l'entreprise qui va permettre de réconcilier certaines exigences contradictoires. D'un point de vue 


\section{L'appropriation de la RSE par les dirigeants de PME. Le réseau comme vecteur de l'apprentissage managérial}

pratique, la RSE devient un objectif de gestion, une réponse légitime pour les partenaires.

Selon ce modèle, le choix de développer telles ou telles pratiques de RSE traduit une maturité plus ou moins grande de l'entreprise face à la notion de RSE et la capacité à s'approprier de manière plus ou moins complète cette notion.

Tableau 1 : Postures managériales face à la RSE (d'après Saulquin \& Schier, 2007)

\begin{tabular}{|c|c|c|c|c|}
\hline $\begin{array}{l}\text { Attitude } \\
\text { face à la } \\
\text { RSE }\end{array}$ & Passive & Réactive & Active & Proactive \\
\hline $\begin{array}{l}\text { Perception } \\
\text { de la RSE }\end{array}$ & $\mathrm{RSE}=$ contrainte & $\begin{array}{l}\text { RSE = levier } \\
\text { d'ouverture }\end{array}$ & $\begin{array}{l}\text { RSE = levier } \\
\text { de dynamique } \\
\text { interne }\end{array}$ & $\begin{array}{l}\text { RSE = levier } \\
\text { stratégique }\end{array}$ \\
\hline $\begin{array}{l}\text { Rôle de la } \\
\text { RSE }\end{array}$ & $\begin{array}{l}\text { Répondre à la } \\
\text { pression des } \\
\text { parties prenantes }\end{array}$ & $\begin{array}{l}\text { Minimiser les } \\
\text { risques }\end{array}$ & Innover & $\begin{array}{l}\text { Innover sur le long } \\
\text { terme pour les } \\
\text { parties prenantes }\end{array}$ \\
\hline $\begin{array}{l}\text { Pratiques } \\
\text { de RSE }\end{array}$ & $\begin{array}{l}\text { L'entreprise attend } \\
\text { que la pression } \\
\text { des PP soit forte } \\
\text { pour mener des } \\
\text { actions. } \\
\text { Exemples : mise } \\
\text { en conformité } \\
\text { légale et } \\
\text { règlementaire, } \\
\text { qualité des } \\
\text { produits, gestion } \\
\text { des déchets... }\end{array}$ & $\begin{array}{l}\text { L'entreprise ne } \\
\text { veut pas que les } \\
\text { risques sociaux et } \\
\text { environnementaux } \\
\text { entachent sa } \\
\text { réputation. } \\
\text { Exemples: } \\
\text { Sponsoring et } \\
\text { activités caritatives, } \\
\text { communication } \\
\text { environnementale, } \\
\text { recrutements } \\
\text { locaux... }\end{array}$ & $\begin{array}{l}\text { Opportunité } \\
\text { pour trouver } \\
\text { de nouveaux } \\
\text { produits et } \\
\text { services et avoir } \\
\text { des démarches } \\
\text { innovantes. } \\
\text { Exemples : Eco } \\
\text { conception, } \\
\text { promotion de } \\
\text { la diversité, } \\
\text { mécanismes de } \\
\text { motivation des } \\
\text { RH... }\end{array}$ & $\begin{array}{l}\text { Politiques de RSE } \\
\text { définies pour les } \\
\text { PP. } \\
\text { Exemples : Co } \\
\text { construction des } \\
\text { offres et des } \\
\text { solutions, durabilité } \\
\text { des produits, } \\
\text { procédures de } \\
\text { gestion de crises... }\end{array}$ \\
\hline Posture & Mécaniste & $\begin{array}{l}\text { Opportuniste \& } \\
\text { Cosmétique }\end{array}$ & Processuelle & Engagée \\
\hline
\end{tabular}

Même si la théorie des parties prenantes permet de prendre en considération l'environnement de l'entreprise, elle est de nature statique et ne permet pas de saisir les processus dynamiques d'appropriation de la RSE et de gouvernance. Elle se focalise essentiellement sur les différentes parties prenantes, leurs attentes, leurs rentes et les conflits d'intérêt qui en découlent. Elle considère encore les différentes parties prenantes de manière indépendante les unes par rapport aux autres. Chacune est finalement rémunérée selon sa rareté et sa contribution à la création de valeur. Charreaux et Desbrières (1998) nous expliquent que chaque partie prenante ne va s'impliquer que dans la mesure où elle perçoit une partie de la richesse créée. Cette lecture peut induire, selon Baret (2007), un management " écartelé " qui vise à satisfaire des exigences contradictoires, et ce faisant néglige les interactions constantes entre les différents partenaires. Pour l'auteur, « ces dernières font 'système' avec l'entreprise, via d'incessants 
feedbacks qui interfèrent entre eux. Le risque est, alors, d'avoir une vision figée de l'organisation qui ne permet pas de comprendre comment elle s'approprie les objectifs sociétaux ». Cette théorie semble en définitive insuffisante pour expliquer comment les décideurs dans une entreprise sont susceptibles d'intégrer les enjeux de la RSE. II subsiste de nombreuses questions sans réponse : qui est reconnu comme partie prenante par les managers ? Qui représente ces parties prenantes face au dirigeant dans les conflits d'intérêt ? Jusqu'où aller avec les parties prenantes et quelle part du pouvoir le dirigeant est-il disposé à leur céder? Quel type de relation nouer avec les parties prenantes et quelle place leur réserver?

Pour essayer de comprendre les aspects dynamiques de l'appropriation de la RSE, nous proposons de mobiliser un autre cadre théorique, celui de l'apprentissage managérial, qui permet de restituer l'introduction des valeurs sociétales dans les PME notamment.

\section{Le réseau comme vecteur d'apprentissage managérial}

En matière de RSE, beaucoup de travaux ont montré le rôle déterminant de la complémentarité entre les compétences internes des entreprises et les dispositifs de coordination mis en œuvre au plan local (Fort et al., 2005). Ainsi, Postner et Schmidt (1984); Harte et Owen (1987) soulignent le rôle des acteurs de proximité et leur prise en compte dans la stratégie de l'entreprise.

\subsection{La démarche collective favorisée par l'ancrage territorial}

De nombreux travaux se sont efforcés d'analyser les liens de l'entreprise avec son milieu social (Bertrand, 1999 ; Julien, 1996). Ils nous permettent d'établir que l'ancrage territorial et la proximité facilitent le partenariat entre entreprises et collectivités locales ayant pour objectif le développement économique régional (Barabel et Alii, 2004).

Ainsi, pour pallier le manque de moyens humains et financiers qui caractérise la plupart des PME, de nombreux programmes et aides sont proposés en France par les Chambres de Commerce et d'Industrie $(\mathrm{CCl})$ :

- des programmes collectifs visant à la prise en compte du développement durable. Ces actions permettent de mutualiser les coûts pour l'entreprise et de bénéficier d'une dynamique de groupe favorisant les échanges de bonnes pratiques ;

- l'organisation de séances d'informations, de sensibilisation et de formations par les $\mathrm{CCl}$ ou l'animation de clubs d'entreprises sur le thème du développement durable ;

- l'appui à la création d'entreprises et au développement économique dans les filières liées au développement durable ; 


\section{L'appropriation de la RSE par les dirigeants de PME. Le réseau comme vecteur de l'apprentissage managérial}

- des diagnostics thématiques (environnement, sécurité, RH...) pour faire le point sur la situation des PME.

Des actions de ce type ont été menées avec succès et participent à la dynamique durable du tissu économique de la région Centre. Le magazine L'Entreprise ${ }^{67}$ place en effet l'attractivité économique de l'agglomération de Tours au second rang national pour les agglomérations de 200.000 à 500.000 habitants. Réalisé à partir de critères objectifs (population, nombre d'étudiants, niveau de revenu moyen des foyers, infrastructures de transport, marché immobilier, fiscalité locale...) et des données relatives aux entreprises (nombre d'entreprises pour 1.000 habitants, taux de création, de défaillance, chiffre d'affaires et rentabilité...), ce classement présente une photographie du dynamisme économique des villes et de leur capacité à attirer sur leur territoire de nouvelles entreprises.

En 2006, l'Assemblée des Chambres Françaises de Commerce et d'Industrie (ACFCl) a mené une enquête sur la prise en compte du développement durable par les PME. Cette étude montre que les dirigeants intègrent de plus en plus de démarches responsables dans leurs entreprises. Ces démarches sont souvent initiées soit par mimétisme, soit par la mobilisation d'une structure d'appui ayant compris l'enjeu de concilier rentabilité et responsabilité. Tel est le cas du Club des Jeunes Dirigeants (CJD), groupement de chefs d'entreprise et de cadres dirigeants engagés dans la mise en œuvre d'un libéralisme responsable. Cette organisation a notamment élaboré, en 2004, un référentiel à destination des dirigeants de PME souhaitant intégrer des démarches responsables.

Les différents outils de management de la RSE destinés aux entreprises, et plus particulièrement aux PME sont avant tout le résultat d'un travail mené par des dirigeants d'entreprises organisés en réseau, ayant les mêmes préoccupations d'innover et d'améliorer la performance de l'entreprise, tout en respectant les valeurs sociales et environnementales du travail. Les relations réticulaires favoriseraient par conséquent la coopération entre entreprises et leur développement mutuel.

\subsection{Le réseau comme clé de lecture de l'apprentissage managérial du dirigeant de PME}

Représentant près de $90 \%$ du tissu économique français en nombre d'entreprises et constituant de ce fait un enjeu de compétitivité important, les PME en tant qu'objet d'analyse justifient les études qui leur sont consacrées. La recherche en PME permet de faire apparaître " concrètement, et visiblement aux yeux de l'observateur, ce qui est caché, difficile à saisir et à interpréter dans les organisations de grande dimension " (Marchesnay, 1993). Le phénomène observé pourrait donc être plus facilement appréhendable par l'observateur.

67. L’Entreprise, octobre $2007, n^{\circ} 260$ 
Certains auteurs comme Capron et Quairel-Lanoizelée (2004) ou encore Lapointe et Gendron (2004) semblent s'accorder sur la pertinence d'une démarche particulière de RSE dans l'univers des PME par référence aux spécificités de ces organisations par ailleurs largement étudiées (Julien et Marchesnay, 1996 ; Torrès, 1999) :

- le rôle fondamental du propriétaire-dirigeant, qui irrigue la totalité du fonctionnement tant interne qu'externe de l'entreprise (Marchesnay et Fourcade, 1997), engendrant une forte personnalisation de la gestion des PME ;

- une stratégie souvent implicite, à court-terme et basée sur la vision du dirigeant ;

- une organisation fondée sur la proximité, que ce soit au sein de l'entreprise ou dans les relations avec les clients, fournisseurs et autres partenaires (Torrès, 1999). Le dirigeant joue un rôle majeur dans la mobilisation de réseaux d'influence.

Enfin, ce type d'entreprise présente une insertion territoriale forte. Comme l'ont montré de nombreuses études empiriques (Fourcade et Torrès, 2003), l'organisation et la qualité du milieu économique local constituent des facteurs de réussite du développement et de la performance des PME. Lorsque tous les acteurs territoriaux s'engagent dans un processus de coopération durable, une dynamique se crée et renforce l'attractivité des entreprises (Capiez, 2007).

Pacitto et Tordjman (1999) ont démontré que le mode d'apprentissage privilégié des dirigeants de PME/PMl est l'interaction à travers l'insertion dans des réseaux d'acteurs. II apparaît que la capacité du dirigeant à s'approprier et à intégrer de nouveaux savoirs est fondamentale dans le processus d'innovation. Ainsi, ce dernier apparaît comme " un processus d'apprentissage où les capacités internes et externes de l'entreprise se combinent dans des interactions avec son environnement » (Nicolas et Hy, 2000).

La capacité d'apprentissage des dirigeants se manifeste par leur aptitude à acquérir de l'information dans le cadre d'interactions avec les parties prenantes, notamment grâce à l'insertion dans des réseaux d'acteurs. L'appropriation et le traitement de cette information constituent également deux éléments déterminants de l'apprentissage managérial. Ainsi, pour mettre en place des outils de gestion de la RSE innovants, les dirigeants de PME vont être proactifs dans la recherche d'informations ou dans la participation à des démarches collectives le plus souvent animées par leurs fédérations professionnelles, associations ou groupements économiques.

Peillon (2005) définit le groupement comme « s'agissant de plusieurs PME qui se regroupent afin de mener à bien un projet, tout en restant juridiquement indépendantes ". Cette définition laisse entrevoir l'extrême diversité du 


\section{L'appropriation de la RSE par les dirigeants de PME. Le réseau comme vecteur de l'apprentissage managérial}

phénomène: le groupement de PME est, tout comme la coopération interentreprises, un phénomène à la fois singulier et multiforme. Les formes prises par l'accord, les objectifs visés et les PME concernées varient considérablement selon les cas. Selon Heitz et Douard (2003), les coopérations interentreprises s'entendent au sens « de l'interaction plus ou moins étendue entre les activités de deux ou plusieurs entreprises juridiquement distinctes ».

Bien que de nombreux travaux existent sur la coopération entre les petites entreprises, notamment au sein des districts industriels ou des systèmes productifs locaux, il n'existe pas de littérature sur les groupements de PME (Peillon, 2005). Ainsi, la définition la plus appropriée pour aborder le groupement de PME est celle du réseau. Heitz et Douard (2003) caractérisent le réseau dans le sens d'une généralisation plus grande, c'est-à-dire « en tant qu'objet d'analyse global de l'interaction d'au moins deux entreprises juridiquement distinctes ; il se caractérise par des critères variés, tels que le nombre d'entreprises en interaction, la nature des interrelations, le niveau de contractualisation des engagements, les actifs propres au réseau, etc. ".

La notion de réseau peut être abordée d'après deux conceptions opposées. Alors que Williamson $(1975,1991)$ définit le réseau comme une forme hybride entre marché et hiérarchie, Powell (1990) montre qu'il est un mode d'échange possédant sa logique propre basée sur la réciprocité, la collaboration, la complémentarité, la réputation et la communication. C'est cette deuxième conception que nous privilégions pour appréhender les réseaux de PME, considérés par Donckels et Lambrecht (1997) comme des systèmes organisés de relations dontl'entrepreneur est l'acteur clé. Quairel et Auberger (2005) affirment que l'implantation territoriale est fondamentale pour les dirigeants de PME. En raison de leurs relations de proximité, les réseaux régionaux pourraient favoriser l'appropriation de démarches de RSE dans les PME.

Ainsi, si l'on considère le point de vue du dirigeant d'une PME, les acteurs avec lesquels il est en relation sont souvent les parties prenantes territoriales. L'analyse du réseau par le dirigeant consiste donc à repérer les acteurs clés internes et externes, comprendre et apprendre le mode de fonctionnement du réseau, se familiariser avec les codes de conduite existants et mesurer l'efficience de l'organisation réticulaire. Comme le note Veltz (2002), « faire partie d'un réseau, d'un tissu dynamique permet d'être dans le coup, de savoir ce qui n'est écrit nulle part, même dans la presse spécialisée, de connaître les réputations des fournisseurs et des clients. Pour un chef d'entreprise, la capacité de jugement [...] est fondamentale. Or, ce jugement est infiniment plus difficile pour l'entrepreneur isolé, débutant, socialement enclavé, que pour l'entrepreneur inséré dans un milieu ».

Le réseau est par conséquent un cadre dans lequel les ressources et les capacités s'agencent entre les acteurs internes et externes. Economiquement, le réseau 
apparaît comme un ensemble de moyens matériels et immatériels permettant aux parties prenantes d'établir des relations spécifiques créatrices de valeur par la synergie et l'apprentissage managérial dégagés, pour lesquels l'implantation territoriale est dominante et l'investissement collectif primordial.

\section{Le rôle du GEIDA dans l'appropriation de démarches responsables}

Après avoir exposé le rôle du réseau dans la mise en place de la RSE, nous proposons d'illustrer nos propos avec une étude qui a pour objet de cerner l'influence du réseau sur l'appropriation de la RSE par des dirigeants de PME. Nous aborderons successivement les considérations méthodologiques et le contexte de notre étude concernant le Groupement des Entreprises du Val d'Amboise en Indre et Loire (GEIDA).

Une première collecte des données s'est effectuée de mai à septembre 2007 par entretiens individuels semi-directifs d'une durée moyenne d'une heure auprès d'une vingtaine de dirigeants de PME/PMI et de consultants spécialisés dans le développement durable. Les responsables qualité, environnement ou ressources humaines sont volontairement exclus du processus de recueil des données dans la mesure où nous avons privilégié les entretiens avec la personne à l'origine de l'impulsion stratégique d'un engagement en faveur du développement durable : le dirigeant de l'entreprise.

II ressort de l'analyse des entretiens exploratoires que la majorité des dirigeants de PME s'adresse à leur représentant professionnel (fédération, association ou groupement) pour obtenir des informations spécifiques sur des thématiques liées au développement durable. De manière générale le représentant professionnel, et plus particulièrement le groupement est considéré par ces dirigeants comme un des relais entre les entreprises et les organismes territoriaux.

Parmi les neuf groupements du département d'Indre-et-Loire, nous focalisons notre attention sur l'étude d'un cas unique, celui du Groupement des Entreprises du Val d'Amboise (GEIDA). Ce groupement est le plus important en termes d'entreprises adhérentes. II rassemble actuellement plus de 70 entreprises implantées sur les différentes zones industrielles qui se sont développées autour de la ville d'Amboise.

Dans un second temps, nous avons mené un entretien avec le Président du GEIDA et le Responsable de la Commission des Ressources Humaines, tous deux chefs d'entreprise. Cet entretien a été conduit à l'aide d'un guide d'entretien complété par les remarques obtenues précédemment. Cette grille de lecture nous permet ainsi d'explorer le lien existant entre la participation à un groupement d'entreprises et l'appropriation de démarches de RSE. 


\section{L'appropriation de la RSE par les dirigeants de PME. Le réseau comme vecteur de l'apprentissage managérial}

\subsection{Présentation du GEIDA}

Le GEIDA se fixe pour but de regrouper les entreprises d'un même secteur géographique, de permettre une meilleure connaissance entre ses membres, d'animer par des actions communes les différents secteurs d'activités et de développer les connaissances de ceux qui la composent, indépendamment de toute représentation politique, religieuse ou philosophique.

Le GEIDAest constitué de huit commissions ${ }^{68}$ dont les membres sont généralement des dirigeants d'entreprise. Pour chaque commission, des réunions de réflexion sont établies afin d'analyser les demandes, rechercher des stratégies et faire naître des thématiques et des solutions intéressant le plus grand nombre. Ensuite, des actions sont mises en œuvre pour répondre aux problématiques collectives. Ces commissions abordent les principes de la Triple Bottom Line ${ }^{69}$ : social, environnemental et économique.

Les plus actives, en termes de communication et de supports aux entreprises, sont les commissions Environnement, Ressources Humaines et Achat.

Encadré 1 : Exemples de démarches responsables impulsées par le GEIDA

Le GEIDA, très actif dans le domaine environnemental, a notamment piloté une opération
collective de gestion des déchets industriels banals ou non dangereux (DIB) en collaboration
avec la Chambre de Commerce et d'Industrie de Touraine. En matière de gestion des Ressources
Humaines, le GEIDA a travaillé sur l'employabilité et la mise en place de formations sur la gestion
des compétences, avec des journées d'accompagnement pour les dirigeants. Enfin, en ce qui
concerne les achats, le groupement fait des appels d'offres et a récemment permis de mettre en
place des tarifs privilégiés d'achat de fournitures de bureau. II s'est également associé en 2006 à
la CCI de Touraine dans un projet intitulé 'MAltrisez Vos Achats' de matières premières (MAIVA).
Actuellement, la commission Achat travaille sur les EPI (Equipements et Protection Individuelle).

Le GEIDA a souhaité travailler plus particulièrement sur des problématiques de GRH telles que les métiers en tension, les difficultés de recrutement, la sensibilisation à la gestion prévisionnelle des emplois et des compétences (GPEC), pour les entreprises situées sur le territoire du Val d'Amboise.

Débuté en janvier 2006, ce projet a été présenté à l'ensemble des entreprises adhérentes au GEIDA. Une enquête a ensuite été soumise, sur ces thématiques, aux chefs d'entreprise et aux responsables des Ressources Humaines.

Les résultats ont confirmé certaines problématiques et inquiétudes liées à la pénurie de main d'œuvre qualifiée pour certaines activités. L'enquête a également révélé le besoin urgent d'une gestion de la pyramide des âges, notamment au regard des futurs départs en retraite. Enfin, le transfert des compétences est

68. Les huit commissions sont les suivantes : Achat, Communication, Contrat de Pays, Economique, Foyers des jeunes travailleurs, Médecine du travail, Ressources Humaines et Environnement.

69. La Triple Bottom Line est la transposition de la notion de développement durable en entreprise par l'évaluation de la performance de l'entreprise sous trois angles : social, environnemental et économique. 
encore trop peu formalisé, l'appréciation des performances est relativement opaque et les dispositifs de formation existants sont encore trop méconnus.

Les membres du Bureau et de la Commission Ressources Humaines du GEIDA ont donc souhaité proposer des actions pouvant répondre aux besoins exprimés par les entreprises:

- Permettre aux entreprises du GEIDA de mieux caractériser leurs offres d'emploi ;

- Favoriser la rencontre entre les chefs d'entreprise du groupement et les demandeurs d'emploi du bassin, ainsi que les jeunes en formation initiale ;

- Valoriser les métiers dont les entreprises ont besoin ;

- Permettre à des demandeurs d'emploi d'accéder aux qualifications recherchées par les entreprises et aux emplois qu'elles proposent ;

- Rapprocher les besoins des entreprises de ce que peuvent proposer les services publics (ANPE...).

Cette démarche illustre l'objectif du GEIDA qui consiste à accompagner ses adhérents dans l'intégration des enjeux sociétaux, en prenant en charge la mise en place de l'enquête susnommée.

Dans ce contexte, Actiforces, organisme de formation professionnelle, a été sollicité pour accompagner ce projet par la mise à disposition de son offre de formation d'actions collectives. Ce projet a bien été accepté par les dirigeants qui ont intégré facilement les actions proposées par l'organisme de formation. Le groupement a prolongé son action de GPEC par une formation à l'entretien professionnel. Par ailleurs, une convention a été signée le 03 décembre 2007 par le GEIDA et la Mission locale pour promouvoir l'insertion professionnelle des jeunes sur les métiers porteurs des zones d'emploi.

La participation aux différentes commissions de réflexion et les échanges avec Actiforces ont permis aux dirigeants de communiquer sur leurs expériences respectives, favorisant ainsi le transfert de connaissances et de compétences, ce qui représente un apport important dans la prise de conscience et la mise en place de démarches de RSE.

\subsection{Analyse des résultats}

Ancrées dans l'action, les dirigeants de PME n'ont bien souvent ni le temps, ni les moyens, ni même l'organisation nécessaire pour s'impliquer pleinement dans la recherche d'informations. L'analyse des entretiens souligne le fait que la mobilisation de ce groupement permet aux dirigeants de neutraliser pour partie l'asymétrie d'informations à laquelle ils se trouvent confrontés. Bénéficiant ainsi d'une information plus concrète en matière de RSE, ces derniers se représentent plus facilement les actions pouvant être intégrées dans la stratégie. 


\section{L'appropriation de la RSE par les dirigeants de PME. Le réseau comme vecteur de l'apprentissage managérial}

Partisan de réunions peu nombreuses mais efficaces, la force du GEIDA réside dans la dynamique de son Bureau, dans la fidélité de ses membres et dans le nombre d'entreprises qu'il représente, dont le chiffre n'a jamais cessé de croître. En tant que gestionnaire du réseau, le GEIDA organise donc des réunions adaptées aux problématiques des dirigeants d'entreprise, ce qui permet de formuler l'hypothèse d'une volonté de rapprochement entre les adhérents et, par conséquent, un processus d'apprentissage managérial.

Des démarches collectives et des actions concrètes sont régulièrement proposées aux entreprises adhérentes qui ont la possibilité de les intégrer dans leur stratégie. Cette structure d'appui facilite la connexion et la coopération entre les entreprises et les institutions spécialisées. Si le GEIDA s'est imposé comme un relais efficace entre les entreprises et les instances territoriales, c'est que bon nombre des dirigeants se sont appropriés les actions concrètes élaborées par ce groupement. L'effet de réseau permet donc une coordination des actions et une mutualisation des compétences et des ressources.

De plus, le GEIDA est parfaitement impliqué dans la vie locale et entretient des contacts réguliers avec de nombreux acteurs territoriaux : la Chambre de Commerce et d'Industrie de Touraine (CCIT), la Direction Départementale du Travail, de l'Emploi et de la Formation Permanente (DDTEFP), la Direction Régionale de l'Industrie, de la Recherche et de l'Environnement (DRIRE), la mairie, les lycées et collèges d'Amboise et diverses associations locales économiques et sociales. Le GEIDA est ainsi perçu comme l'interlocuteur des différentes parties prenantes qu'il sollicite pour intervenir auprès des entreprises. Ce groupement économique a créé un mode d'échange fondé sur la confiance, celle-ci « jouant un rôle essentiel en tant que garant de la transaction et conditionnant la performance du réseau » (Capiez, 2007), au sein duquel l'apprentissage managérial y est favorisé.

Il est donc plus aisé de comprendre comment le GEIDA est devenu un nouvel acteur répondant à l'évolution des frontières de l'entreprise. En s'appuyant sur les compétences et les savoir-faire de chacun de ses membres et des acteurs externes, ce groupement cherche à développer les bonnes pratiques, l'expérimentation et l'innovation pour le bénéfice de tous et ce, par l'échange d'expériences, de connaissances et de ressources tant matérielles qu'immatérielles.

L'élaboration d'une stratégie commune, reposant sur un projet partagé, celui de dynamiser le tissu économique local, facilite la synchronisation entre structures et permet une meilleure gestion de l'autonomie des acteurs (Zardet et al., 2007).

Le GEIDA a su mettre en œuvre une stratégie collective, réguler les relations entre les différents acteurs et répondre ainsi aux attentes sociétales pour une plus grande responsabilisation des entreprises. Le GEIDA dispose donc de la légitimité nécessaire pour piloter et animer des acteurs, leur permettant ainsi de se rapprocher. 
II ne s'agit que des premières conclusions de nos travaux sur l'intégration de pratiques de RSE dans les PME bénéficiant des ressources d'un réseau. Cette discussion nous a permis de mettre en évidence que les membres du GEIDA se sont progressivement appropriés les démarches de RSE initiées par les commissions de réflexion.

Cette étude mériterait d'être réalisée auprès des représentants des autres groupements d'entreprises de la région afin de comparer les résultats. Cette étude pourrait également porter sur l'identification des compétences dont une structure d'appui doit se doter en priorité pour intervenir de façon efficace auprès des PME.

\section{Conclusion}

Les relations réticulaires représentent une stratégie de recherche pour le management de la RSE, celles-ci provoquant des changements de comportements dans les interactions avec les partenaires et dans les processus de communication visant à intégrer les préoccupations de développement durable et de responsabilité sociale. L'analyse des discours nous a permis de mettre en évidence une asymétrie d'informations entre les entreprises et les acteurs encourageant le développement de pratiques de RSE sur le territoire régional. De manière générale, les groupements d'entreprises, les fédérations professionnelles et les experts (consultants, consulaires) semblent avoir pris conscience de cet enjeu. C'est pourquoi ils mettent en place un certain nombre d'actions - démarches collectives ou réunions d'informations - visant à apporter une réponse à leurs adhérents ou clients.

La légitimité d'un réseau et la confiance qu'il sait instaurer auprès des acteurs internes et externes au réseau lui permet de développer les interactions et interdépendances nécessaires à toute coopération entre acteurs d'organisations différentes. Les entreprises bénéficient d'une meilleure connaissance du tissu économique régional, ainsi que d'une meilleure anticipation de leurs besoins en infrastructures et en ressources humaines. Les échanges favorisent l'intégration des préoccupations du développement durable et diminuent l'asymétrie d'informations. Les entreprises appréhendent mieux les attentes de la communauté (les communes notamment) sur les retombées économiques et sociales, se sentent co-responsables de l'espace et sont plus disposées à s'impliquer dans la gestion du territoire. L'organisation en réseau peut donc être considérée comme un nouveau mode de diffusion de la RSE, dépassant les frontières de l'entreprise.

Au-delà de cet exemple, cet article nous a permis de mettre en exergue la posture du dirigeant de PME face à la RSE et de nous interroger sur les apports de la théorie des parties prenantes pour comprendre l'appropriation de la RSE 


\section{L'appropriation de la RSE par les dirigeants de PME. Le réseau comme vecteur de l'apprentissage managérial}

par le manager. II nous apparait que cet éclairage théorique est limité. S'il permet d'intégrer les parties prenantes et leurs attentes, il peine à expliquer les dynamiques d'appropriation des enjeux sociétaux. D'autres grilles de lecture sont alors nécessaires, l'apprentissage réticulaire en est une illustration.

\section{Bibliographie}

Abrams F.W. (1951), " Management Responsibilities in a Complex World », Harvard Business Review, Vol. 29, No. 5, pp.29-34

Allouche J., Huault I., Schmidt G. (2004), « Responsabilité sociale des entreprises : la mesure détournée ? ", 15ème Congrès annuel de l'AGRH, Montréal. Septembre.

Barabel M., Meier O., Schier G. (2004), « Construire le projet stratégique d'un territoire », dans Management local et réseaux d'entreprises, Economica, Paris, pp. 189-207

BaretP.(2007), «Comprendrel'appropriation de la RSE : quel(s)éclairage(s)théorique(s)? »,

XVlème Conférence Internationale de Management Stratégique, AIMS, Montréal, 6-9 juin

Berger-Douce S. (2008), "Rentabilité et pratiques de RSE en milieu PME. Premiers résultats d'une étude française », Management \& Avenir, n¹5, p. 9-29, janvier.

Bertrand N. (1999), « Des stratégies aux comportements spatiaux de l'entreprise : l'insertion locale des PME », Revue internationale PME, vol. 12, n 1-2, pp. 85-106

Bowen H.R. (1953), Social Responsibilities of the Businessman, Harpet \& Row.

Capiez A. (2007), « Réseaux d'entreprises et performance », Revue internationale PME, vol. $20, n^{\circ} 1$, pp. 41-67

Capron M. et Quairel-Lanoizelée F. (2004), Mythes et réalités de l'entreprise responsable - Acteurs, Enjeux et Stratégies, Editions La Découverte, Paris

Caroll A.B. (1979), "AThree dimensional conceptual model of corporate social performance", Academy of Management Review, Vol. 4, pp 497-505.

Charreaux G., Desbrières P. (1998), « Gouvernance des entreprises : valeur partenariale contre valeur actionnariale », Finance, Contrôle, Stratégie, Vol. 1, No. 2, pp. 57-88

Clarkson M.B. (1995), « A stakeholder framework for analyzing and evaluating corporate social performance », Academy of Management Review, Vol. 20, No. 1, pp. 92-117

Damak-Ayadi S., Pesqueux Y. (2003), « Stakeholder Theory in Perspective », Colloque AIMS « Développement durable et entreprise ».

Donckels R., Lambrecht J. (1997), « The network position of small businesses: an exploratory model », Journal of Small Business Management, vol. 35, April, pp. 65-97

Douard J.-P., Heitz M. (2003), « Une lecture des réseaux d'entreprises : prise en compte des formes et des évolutions ", Revue Française de Gestion, vol. 29, n¹46, pp. 23-41

Fort F., Rastoin J.-L., Temri L. (2005), « Les déterminants de l'innovation dans les petites et moyennes entreprises agroalimentaires ", Revue internationale PME, vol. 18, $n^{\circ} 1, p p$. 47-72

Fourcade C., Marchesnay M. (1997), Gestion de la PME/PMI, Editions Nathan, Paris

Fourcade C., Torrès O. (2003), "Les PME en région et mondialisation : processus de 'glocalisation' et dynamique de proximité », Les Cahiers de l'ERFI, vol. 10, n4, pp. 5-26 E. Freeman (1984), Strategic management : a stakeholder approach, Pitman (Harper and Row), Boston

Gond J.P., Mercier S. (2005), « Les théories des parties prenantes : une synthèse critique de la littérature », Les Notes du LIHRE, $n^{\circ}$ 411, pp. 1-22

Harte G.F., Owen D.L. (1987), "Accounting as reality construction: the role of local government social audits", Accounting, Organizations and Society, vol. 12, n², pp. 123141

Igalens J. (2009), Symposium « La responsabilité sociale », Gème Congrès de l'ADERSE, Pau, 22 janvier 
Julien P.-A. (1996), « Entrepreneuriat, développement du territoire et appropriation de l'information », Revue internationale PME, vol. 9, n 3-4, pp. 149-17

Julien P.-A., Marchesnay M. (1996), L'Entrepreneuriat, Economica, Collection Gestion, Paris

Luetkenhorst W. (2004), "Corporate Social Responsibility and the Development Agenda - A Case for Actively Involving Small and Medium Enterprises", Intereconomics, mai-juin, pp. 157-166

Marchesnay M. (1993), «PME, stratégie et recherche », Revue Française de Gestion, n 95, pp.70-76

Mitchell R.K., Agle B.R., Wood D.J. (1997), "Towards a theory of stakeholder identification and salience: defining who and what really counts", Academy of management Review, 22(4), p.853-886.

Nicolas F., Hy M. (2000), «Apprentissage technologique et innovation en agro-alimentaire », Economie rurale, $n^{\circ} 257$, pp. $27-41$

Pacitto J.C., Tordjman F. (1999), « L'innovation technologique dans la très petite entreprise industrielle française : ce que disent les statistiques », Revue internationale PME, vol. 12, n³, pp. 59-90

S. Peillon (2005), "Une analyse dynamique du pilotage des groupements de PME », Revue internationale PME, vol. 18, n¹, pp. 103-128

Pierre X., Zardet V. (2007), « Distance spatiale et cognitive entre les acteurs impliqués dans le management du territoire », Actes du Colloque « Loin, proche : la dimension spatiale dans le management des organisations », Orléans

Posner B.Z., Schmidt W.H. (1984), "Values and the American Manager: an Update", California Management Review, vol. 26, n³, pp. 202-216

Powell W.W. (1990), « Neither market nor hierarchy: networks forms of organisation », dans Cummings L., Staw B. (dir.), Research in Organizational Behavior, vol. 12, Greenwich, CT, JAI Press, pp. 295-336

Quairel F., Auberger M.N. (2005), « Management responsable et PME : une relecture du concept de responsabilité sociétale de l'entreprise ", Revue des Sciences de Gestion, Direction et Gestion, ${ }^{\circ}$ 211-212, pp. 111- 126

Saulquin JY., Schier G. (2005), « La RSE comme obligation/occasion de revisiter le concept de performance », Congrès GREFIGE, Nancy, 17 \& 18 mars.

Saulquin JY., Schier G. (2005), « Typologie des pratiques de RSE », Colloque AIMS/ISIAM/ IAE Nancy 2, GREFIGE-CEREMO, Agadir, avril

Torrès O. (1999), Les PME, Editions Flammarion, Collection Dominos, Paris

Veltz P. (2002), Des lieux et des liens : le territoire français à l'heure de la mondialisation, Editions de l'Aube, Paris

Wartick S., Cochran P. (1985), "The evolution of the Corporate Social Performance Model », Academy of Management Review, Vol. 10, No. 1, pp. 166-179

Williamson O. (1975), Market and Hierarchy: Analyses and Antitrust Implications, The Free Press, New-York

Williamson O. (1991), "Strategizing, economizing and economic organization", Strategic Management Journal, vol. 12, pp. 75-94 